\title{
BACK TO BASICS ON LEADERSHIP IN AN EMERGENT ECONOMY
}

\author{
DOI: 10.17261/Pressacademia.2019.1052 \\ RJBM-V.6-ISS.2-2019(3)-p.121-136
}

Muberra Yuksel ${ }^{1}$, Feyza Oran ${ }^{2}$

${ }^{1}$ Kadir Has University, Faculty of Applied Sciences, Department of International Trade and Logistics, Istanbul, Turkey. muberray@khas.edu.tr, ORCID: https://orcid.org/0000-0002-1838-2655

${ }^{2}$ Kadir Has Vocational School, Department of Logistics, Istanbul, Turkey.

feyza.oran@khas.edu.tr, ORCID: https://orcid.org/0000-0003-4527-0015

Date Received: May 20, 2019

Date Accepted: June 25, 2019

To cite this document

Yuksel, M., Oran, F., (2019). Back to basics on leadership in an emergent economy. Research Journal of Business and Management (RJBM),

V.6(2), p.121-136

Permemant link to this document: http://doi.org/10.17261/Pressacademia.2019.1052

Copyright: Published by PressAcademia and limited licenced re-use rights only.

\section{ABSTRACT}

Purpose- Leadership styles such as delegative, democratic, autocratic, and ethical leadership with respect to various aspects of trust and their causal relations along with determining the salient leadership style at public schools are the purpose of this research. Our assumption is that organizational trust is at the crux of human resource management since a healthy relationship between leaders and employees is a prerequisite for open communication, knowledge sharing and conflict management.

Methodology- Our research question is to understand the basic relationships between leadership styles and trust between principals and teachers. We are conducting a survey at designated school sites. Our questionnaire is comprised of three sections: Socio-demographic variables, leadership styles and organizational trust.

Findings- We have employed structural equation modelling (SEM) and found significant relationship between leadership styles and trust and vice versa. In sum, managerial trust has a significant effect on leadership styles which reveals the fact that dyadic relationships between leaders and employees enhance mutual trust.

Conclusion- In sum, managerial trust has a significant effect on leadership styles which reveals the fact that dyadic relationships between leaders and employees enhance mutual trust. Such relationships need to be considered in human resource management (SHRM).

Keywords: Leadership theories, SHRM, SEM, trust and competencies.

JEL Codes: M10, M12, M19.

\section{INTRODUCTION}

We are living in a business epoch of strengthening social networks based on interest and weakening social organizations and communities based on trust. Open communication and sharing problems as well as opportunities support both collaboration and learning, which are indispensable for effective leadership and human resource management at various sectors. Leaders at work and at schools enable their followers to be in a state of flow so that efficiency is attained according to Mihaly Csikszentmihalyi, who is one of the founding fathers of positive psychology (1990). He focuses on leadership competencies with particular emphasis on "flow theory" where flow is:

"A mental state in which a person performing an activity is fully immersed in a feeling of energized focus, full involvement, and enjoyment" (2012).

Csikszentmihalyi's theory of flow focuses mainly on ethical leadership framework, that is derived from well-known basic theories. According to him, among the 29 leadership competencies four are directly linked to his notion of flow: (1) Strategic thinking (setting clear meaningful goals), (2) Applying personal strengths for a common goal (such as self-esteem), (3) Balancing skill and challenge level (focusing on competencies and efforts along with results), and (4) Frequent feedback on performance all of which are relevant to trust, ethical responsibility and understanding of multiple perspectives. 
Lately, leadership development and styles are gaining importance in the process of properly managing human resources in education. In this article, we have used (Ateş, 2005) are derived from Clark (1997 and 2004). We have analyzed ethical, autocratic, democratic and delegative (laissez-faire) leadership with respect to various aspects of trust and socio-demographic characteristics of educators/ teachers.

Organizational trust is the crux of strategic human resource management since a healthy relationship between leaders and employees is a prerequisite for open communication, knowledge sharing, and learning. Competencies which consist of knowledge, attitude and behavior are shaped and prospered in a positively reinforced learning environment. Organizational trust is regarded as a fundamental threshold, since it enhances job security at times of uncertainty and risk (Blomqvist \& Ståhle, 1998, p. 11); further, consistent and expected behavior of leaders also enriches the trust environment.

That is why, leadership style is a significant factor in the formation of organizational and/or managerial trust. However, leadership styles and trust may not enhance predictability, harmony and strategic flexibility in planning and implementation if there is no incremental and/ or systematic development on the whole. Hence, with increasing trust between managers and subordinates, the perceived level of risk increases with uncalculated actions. Blomqvist and Stahle have based their theoretical framework on both interpersonal and managerial trust (1998; 12). We have employed Scott's (1981) and Adams' (2004) scales of organizational trust which has been inspired from Luhmann's framework (1979) and it is adapted by Kanten (2012).

As an emergent economy, Turkish education system is continually changing both its programs as well as its structures. Therefore, principals as well as teachers at both primary and secondary schools have difficulty in adapting and internalizing new priorities. Consequently, particular leadership styles may become more predominant as hands-off policy often followed by principals at times of uncertainty and complexity. Further, organizational trust may have a significant effect on leadership styles, since especially dyadic relationships between leaders and employees enhance mutual trust under such fluid circumstances of uncertainty. We have limited our research to this interactive process between trust and leadership styles selected for the sake of brevity.

\section{LITERATURE REVIEW}

The leadership literature is replete with theoretical frameworks and models, describing different leadership styles. Leadership is the social interaction process within teams through which followers participate voluntarily in decision-making; whereas, leadership style is the pattern of attitudes and behaviors of leaders towards their teams. Lewin, Lippit and White's seminal piece (1939) have first defined three major leadership styles as: autocratic, democratic, and laissez faire/ delegative (Bhatti, Maitlo, Hashmi \& Shaikh, 2012; p. 192-193).

We have employed here Donald Clark's four operationalized leadership styles that includes ethical leadership since it is especially significant within the context of education sector (1997). Clark has made research in middle schools and his questionnaire emphasized the effectiveness of these four leadership styles particularly between school principals and teachers (2004). Since our research is limited to state schools, and education systems have continuously been transformed by governments, it is not possible to employ multifactor leadership questionnaire of modern leadership theories. Besides, operationalization of classical leadership styles is both widely accepted and definitions and demarcation lines between these styles are clear. Below are the definitions of these styles:

$\checkmark \quad$ Democratic leaders have participative style and they tend to include subordinates in all decision-making; therefore, it is time-consuming for some sectors despite the fact that it enhances tolerance and satisfaction (Oshagbemi and Gill, 2003; Bhatti, Maitlo, Shaikh, Hashmi, \& Shaikh, 2012, p. 193),

$\checkmark$ Autocratic leaders have rule-abiding/ bureaucratic and controlling style and they tend to monitor and correct subordinates closely and expect strict obedience (Rast, Hogg, \& Giessner, 2013, p. 636),

$\checkmark$ Delegative (laissez-faire) leaders reveal a yielding and withdrawn style and they tend to avoid conflict and responsibilities, further they tend to hesitate in guidance or feedback. They either avoid being the center of attention or act too late when problems arise (Buch, Martinsen, \& Kuvaas, 2015, p. 117),

$\checkmark \quad$ Ethical leaders walk the talk and integrity, fairness along with trustworthiness of leaders are highlighted. Such leaders act as role models for subordinates who learn and internalize the attitudes and values through social learning theory (Brown, Trevino and Harrison, 2005; Resick, Hargis, Shao, \& Dust, 2013, p. 954). 
Previous research on trust has mostly been ambiguous (Barber, 1983). Most research on trust at the individual, group/ team, and organizational levels of analysis typically suffer from unidimensional conceptualization and operationalization (Barber, 1983; Luhmann, 1979). Luhmann's writings on trust are very well-known and largely cited by researchers in many disciplines. Yet, only the 'early' writings of Luhmann have been widely used. (See Janne Jalava's dissertation for further information on Luhmann's later advanced systems theory and its relation to his early studies of trust).

Following "early" Luhmann, in our model of organizational trust, we have also regarded trust as a decision and/ or a prerequisite of communication and management. According to Luhmann, "to trust is to take a risk (1979; p. 24). Moreover, he distinguishes between familiarity, trust and confidence and three levels of analyses favoring confidence in his later works. Thus, we have only differentiated managerial trust and interpersonal trust, which are both dyadic and mutual and regarded organizational trust as a subsystem simply at these two analysis levels that are operationalized. However, personal trust and interpersonal are not enough to explain the wider processes according to Luhmann.

We have not focused on organizational trust at a higher level or "systemic confidence", that is comprised of programs and codes, since "late" Luhmann's advanced systems theory is beyond the scope of this paper. We have employed Scott's (1981) and Adams' (2004) scales of organizational trust which has been inspired from Luhmann's framework and it is adapted by Kanten (2012). In the below conceptual framework, we have analyzed trust and leadership styles both as dependent and independent variables since they have effect on each other.

Figure 1: Conceptual Model

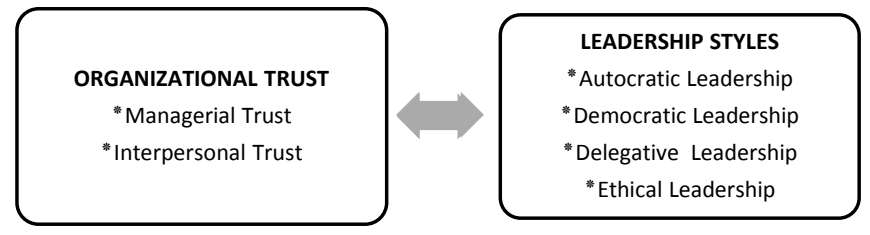

\section{DATA AND METHODOLOGY}

We are conducting a survey at state school sites and our questionnaire is comprised of three sections: Socio-demographic variables, leadership styles and organizational trust. The demographic characteristics that are considered are: age, gender, education, seniority/ tenure track, marital status, hometown (during upbringing of teachers during primary and secondary schooling years between 7-19 of age).

We have conducted a survey and reached 314 teachers and 12 principals and 26 vice principals gathered from 12 different junior high schools and out of 2197 teachers in a district of Istanbul, Turkey. For the sake of brevity, we have used convenience sampling. We analyzed demographic data through descriptive statistics and used explanatory factor analysis using SPSS. We employed AMOS program for SEM.

Demographic features of teachers are demonstrated in Table 1 through descriptive statistics: 
Table 1: Demographic Variables

\begin{tabular}{|c|c|c|c|}
\hline & Group & Frequency & \%Percent \\
\hline \multirow[t]{3}{*}{ Gender } & Female & 191 & 60,8 \\
\hline & Male & 123 & 39,2 \\
\hline & Total & 314 & 100,0 \\
\hline \multirow[t]{3}{*}{ Marital Statues } & Married & 147 & 46,8 \\
\hline & Single & 167 & 53,2 \\
\hline & Total & 314 & 100,0 \\
\hline \multirow[t]{6}{*}{ Seniority } & Less than 1 year & 63 & 20,1 \\
\hline & $1-5$ years & 204 & 65,0 \\
\hline & $6-10$ years & 29 & 9,2 \\
\hline & $11-15$ years & 15 & 4,8 \\
\hline & 21 years and over & 3 & 1,0 \\
\hline & Total & 314 & 100,0 \\
\hline \multirow[t]{5}{*}{ Age } & $20-29$ years & 171 & 54,5 \\
\hline & $30-39$ years & 113 & 36,0 \\
\hline & $40-49$ years & 25 & 8,0 \\
\hline & 50 years and over & 5 & 1,6 \\
\hline & Total & 314 & 100,0 \\
\hline \multirow[t]{6}{*}{ Hometown } & Village & 32 & 10,2 \\
\hline & Sub -district & 15 & 4,8 \\
\hline & Town /District & 83 & 26,4 \\
\hline & Province & 67 & 21,3 \\
\hline & Metropolitan city & 117 & 37,3 \\
\hline & Total & 314 & 100,0 \\
\hline
\end{tabular}

Our major research question is to understand the relationship between the leadership styles and trust as well as determining the salient leadership style in our sample. Our suggested hypotheses are:

1. Ethical leadership has a significant effect on organizational trust or vice versa.

2. Democratic leadership has a significant effect on organizational trust or vice versa.

3. Autocratic leadership has a significant effect on organizational trust or vice versa.

4. Delegative (laissez faire) leadership has a significant effect on organizational trust or vice versa.

In sum, organizational trust - be it managerial or interpersonal - encourages people to take risks and draw lessons from errors. Therefore, trust is a challenge for people to learn and develop their competencies better in trustworthy environments where people are not afraid of the fact that others might take advantage of them. Interpersonal trust has both cognitive and affective components. The cognitive aspect is based upon prior experience and familiarity from the past and it is conditional, while the latter focuses on reciprocal emotional investments for the future. Thus, open and transparent communication, collaboration and teamwork depends on trust, flow, and leadership as suggested by Clark (1997), Csikszentmihalyi (1990) and Luhmann (1979). 


\section{FINDINGS AND DISCUSSIONS}

Below we have summarized the descriptive statistics on scales of leadership styles. 40 Questions with their frequencies are as follows: (See the research questionnaire with 56 questions excluding demographic variables at the Appendix).

Table 2: 40 Survey Questions and their Frequencies

\begin{tabular}{|c|c|c|c|c|c|c|c|c|}
\hline & $\begin{array}{l}\text { Frequency } \\
\text { \%Percent }\end{array}$ & $\begin{array}{l}\text { I strongly } \\
\text { disagree }\end{array}$ & $\begin{array}{l}\text { I do not } \\
\text { agree }\end{array}$ & undecided & I agree & $\begin{array}{c}\text { I strongly } \\
\text { agree }\end{array}$ & Mean & $\begin{array}{c}\text { Std. } \\
\text { Deviation }\end{array}$ \\
\hline \multirow{2}{*}{ Q 1} & Frequency & 59 & 57 & 30 & 109 & 59 & \multirow{2}{*}{3,17} & \multirow{2}{*}{1,418} \\
\hline & Percent & 18,8 & 18,2 & 9,6 & 34,7 & 18,8 & & \\
\hline \multirow{2}{*}{ Q 2} & Frequency & 71 & 59 & 26 & 103 & 55 & \multirow{2}{*}{3,04} & \multirow{2}{*}{1,458} \\
\hline & Percent & 22,6 & 18,8 & 8,3 & 32,8 & 17,5 & & \\
\hline \multirow{2}{*}{ Q 3} & Frequency & 23 & 51 & 34 & 118 & 88 & \multirow{2}{*}{3,63} & \multirow{2}{*}{1,250} \\
\hline & Percent & 7,3 & 16,2 & 10,8 & 37,6 & 28,0 & & \\
\hline \multirow{2}{*}{ Q 4} & Frequency & 58 & 55 & 49 & 91 & 61 & \multirow{2}{*}{3,13} & \multirow{2}{*}{1,403} \\
\hline & Percent & 18,5 & 17,5 & 15,6 & 29,0 & 19,4 & & \\
\hline \multirow{2}{*}{ Q 5} & Frequency & 12 & 17 & 20 & 90 & 175 & \multirow{2}{*}{4,27} & \multirow{2}{*}{1,054} \\
\hline & Percent & 3,8 & 5,4 & 6,4 & 28,7 & 55,7 & & \\
\hline \multirow{2}{*}{ Q 6} & Frequency & 14 & 17 & 22 & 109 & 152 & \multirow{2}{*}{4,17} & \multirow{2}{*}{1,071} \\
\hline & Percent & 4,5 & 5,4 & 7,0 & 34,7 & 48,4 & & \\
\hline \multirow{2}{*}{ Q 7} & Frequency & 13 & 16 & 16 & 91 & 178 & \multirow{2}{*}{4,29} & \multirow{2}{*}{1,055} \\
\hline & Percent & 4,1 & 5,1 & 5,1 & 29,0 & 56,7 & & \\
\hline \multirow{2}{*}{ Q 8} & Frequency & 21 & 29 & 44 & 122 & 98 & \multirow{2}{*}{3,79} & 1176 \\
\hline & Percent & 6,7 & 9,2 & 14,0 & 38,9 & 31,2 & & $1,1 / 0$ \\
\hline & Frequency & 19 & 25 & 26 & 112 & 132 & & \\
\hline Q9 & Percent & 6,1 & 8,0 & 8,3 & 35,7 & 42,0 & 4,00 & $1,1 / 1$ \\
\hline 010 & Frequency & 13 & 23 & 33 & 90 & 155 & 412 & 1120 \\
\hline 40 & Percent & 4,1 & 7,3 & 10,5 & 28,7 & 49,4 & 4,12 & $\perp, \perp \angle U$ \\
\hline 011 & Frequency & 16 & 26 & 42 & 109 & 121 & 393 & 1144 \\
\hline Q 11 & Percent & 5,1 & 8,3 & 13,4 & 34,7 & 38,5 & 3,93 & 1,144 \\
\hline 012 & Frequency & 10 & 11 & 20 & 78 & 195 & & \\
\hline Q 12 & Percent & 3,2 & 3,5 & 6,4 & 24,8 & 62,1 & 4,39 & ,980 \\
\hline 013 & Frequency & 9 & 8 & 15 & 77 & 205 & 447 & 919 \\
\hline & Percent & 2,9 & 2,5 & 4,8 & 24,5 & 65,3 & 4,47 & כערן \\
\hline 014 & Frequency & 13 & 9 & 40 & 85 & 167 & 4.22 & 1049 \\
\hline Q 14 & Percent & 4,1 & 2,9 & 12,7 & 27,1 & 53,2 & $4, \angle 2$ & 1,049 \\
\hline 015 & Frequency & 4 & 20 & 53 & 125 & 112 & בחת & 917 \\
\hline Q 15 & Percent & 1,3 & 6,4 & 16,9 & 39,8 & 35,7 & 4,02 & ,94/ \\
\hline 016 & Frequency & 29 & 25 & 87 & 91 & 82 & 355 & 1220 \\
\hline 4.0 & Percent & 9,2 & 8,0 & 27,7 & 29,0 & 26,1 & כבנה & $1,<<U$ \\
\hline 017 & Frequency & 17 & 15 & 49 & 86 & 147 & 405 & 1142 \\
\hline Q17 & Percent & 5,4 & 4,8 & 15,6 & 27,4 & 46,8 & 4,05 & 1,142 \\
\hline 018 & Frequency & 12 & 17 & 33 & 86 & 166 & 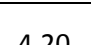 & 1076 \\
\hline Q 18 & Percent & 3,8 & 5,4 & 10,5 & 27,4 & 52,9 & 4,20 & $1,0 / 6$ \\
\hline 019 & Frequency & 10 & 14 & 26 & 81 & 183 & 432 & 1017 \\
\hline 4.5 & Percent & 3,2 & 4,5 & 8,3 & 25,8 & 58,3 & 4,52 & I, \\
\hline 020 & Frequency & 10 & 9 & 22 & 64 & 209 & 4.44 & 972 \\
\hline & Percent & 3,2 & 2,9 & 7,0 & 20,4 & 66,6 & & \\
\hline 11 & Frequency & 10 & 11 & 21 & 77 & 195 & & \\
\hline Q 21 & Percent & 3,2 & 3,5 & 6,7 & 24,5 & 62,1 & 4,39 & 983 \\
\hline Q 22 & Frequency & 12 & 8 & 27 & 80 & 187 & 4,34 & 1,006 \\
\hline
\end{tabular}




\begin{tabular}{|c|c|c|c|c|c|c|c|c|}
\hline & Percent & 3,8 & 2,5 & 8,6 & 25,5 & 59,6 & & \\
\hline \multirow{2}{*}{ Q 23} & Frequency & 25 & 45 & 35 & 113 & 96 & \multirow{2}{*}{3,67} & \multirow{2}{*}{1,266} \\
\hline & Percent & 8,0 & 14,3 & 11,1 & 36,0 & 30,6 & & \\
\hline \multirow{2}{*}{ Q 24} & Frequency & 25 & 42 & 90 & 97 & 60 & \multirow{2}{*}{3,40} & \multirow{2}{*}{1,171} \\
\hline & Percent & 8,0 & 13,4 & 28,7 & 30,9 & 19,1 & & \\
\hline \multirow{2}{*}{ L25 } & Frequency & 13 & 23 & 85 & 110 & 83 & \multirow{2}{*}{3,72} & \multirow{2}{*}{1,062} \\
\hline & Percent & 4,1 & 7,3 & 27,1 & 35,0 & 26,4 & & \\
\hline \multirow{2}{*}{ Q 26} & Frequency & 7 & 23 & 33 & 114 & 137 & \multirow{2}{*}{4,12} & \multirow{2}{*}{1,012} \\
\hline & Percent & 2,2 & 7,3 & 10,5 & 36,3 & 43,6 & & \\
\hline \multirow{2}{*}{ Q27 } & Frequency & 13 & 24 & 34 & 72 & 171 & \multirow{2}{*}{4,16} & \multirow{2}{*}{1,145} \\
\hline & Percent & 4,1 & 7,6 & 10,8 & 22,9 & 54,5 & & \\
\hline \multirow{2}{*}{ Q 28} & Frequency & 83 & 51 & 30 & 83 & 67 & \multirow{2}{*}{3,00} & \multirow{2}{*}{1,531} \\
\hline & Percent & 26,4 & 16,2 & 9,6 & 26,4 & 21,3 & & \\
\hline \multirow{2}{*}{ Q 29} & Frequency & 17 & 20 & 36 & 92 & 149 & \multirow{2}{*}{4,07} & \multirow{2}{*}{1,153} \\
\hline & Percent & 5,4 & 6,4 & 11,5 & 29,3 & 47,5 & & \\
\hline \multirow{2}{*}{ Q 30} & Frequency & 5 & 14 & 18 & 83 & 194 & \multirow{2}{*}{4,42} & \multirow{2}{*}{ 906 } \\
\hline & Percent & 1,6 & 4,5 & 5,7 & 26,4 & 61,8 & & \\
\hline \multirow{2}{*}{ Q 31} & Frequency & 6 & 16 & 33 & 95 & 164 & \multirow{2}{*}{4,26} & 969 \\
\hline & Percent & 1,9 & 5,1 & 10,5 & 30,3 & 52,2 & & \\
\hline 0 32 & Frequency & 23 & 31 & 67 & 102 & 91 & 366 & בח2 1 \\
\hline 432 & Percent & 7,3 & 9,9 & 21,3 & 32,5 & 29,0 & 3,00 & $1, \angle U Z$ \\
\hline 33 & Frequency & 6 & 16 & 47 & 61 & 184 & 428 & 1019 \\
\hline Q 33 & Percent & 1,9 & 5,1 & 15,0 & 19,4 & 58,6 & 4,28 & 1,019 \\
\hline 34 & Frequency & 9 & 19 & 35 & 97 & 154 & 417 & 1037 \\
\hline Q 34 & Percent & 2,9 & 6,1 & 11,1 & 30,9 & 49,0 & $4,1 /$ & $1,03 /$ \\
\hline 035 & Frequency & 13 & 12 & 38 & 94 & 157 & 110 & 1057 \\
\hline Q & Percent & 4,1 & 3,8 & 12,1 & 29,9 & 50,0 & 4,10 & ובכ, \\
\hline 36 & Frequency & 10 & 8 & 29 & 69 & 198 & 439 & 980 \\
\hline$Q 36$ & Percent & 3,2 & 2,5 & 9,2 & 22,0 & 63,1 & 4,39 & ,980 \\
\hline 037 & Frequency & 16 & 15 & 42 & 95 & 146 & 408 & 1116 \\
\hline Q 31 & Percent & 5,1 & 4,8 & 13,4 & 30,3 & 46,5 & 4,08 & 1,116 \\
\hline 30 & Frequency & 11 & 13 & 34 & 102 & 154 & 110 & ברח 10 \\
\hline 400 & Percent & 3,5 & 4,1 & 10,8 & 32,5 & 49,0 & 4,19 & $1,0<2$ \\
\hline 39 & Frequency & 12 & 10 & 44 & 86 & 162 & م204 & 1045 \\
\hline Q 39 & Percent & 3,8 & 3,2 & 14,0 & 27,4 & 51,6 & 4,20 & 1,045 \\
\hline 010 & Frequency & 14 & 14 & 42 & 91 & 153 & & \\
\hline 440 & Percent & 4,5 & 4,5 & 13,4 & 29,0 & 48,7 & 4,13 & 1,090 \\
\hline
\end{tabular}

As may be seen from the above table; the items that have the highest scores are questions 13, 20 and 30. "It is the leader's job to help subordinates find their "passion." has a mean of 4.47", "When there are differences in role expectations, I work with them to resolve the differences." has a mean of 4.44. "As a rule, leaders should allow subordinates to appraise their own work." has a mean of 4.42 .

Likewise, the items that have the lowest scores are questions 28, 2 and 4. "Employees seek mainly job security." has a mean 3.00. "Effective leaders give orders and clarify procedures so that responsibilities are clear." has a mean 3.04 and "As a rule, employees must be given rewards or punishments in order to motivate them to achieve organizational objectives." has a mean of 3.13.

On table 3 are the decriptive statistics on the scale of organizational trust: 
Table 3: Descriptive Statistics of the Trust Scale

\begin{tabular}{|c|c|c|c|c|c|c|c|c|}
\hline & $\begin{array}{l}\text { Frequency } \\
\text { \%Percent }\end{array}$ & $\begin{array}{l}\text { I strongly } \\
\text { disagree }\end{array}$ & $\begin{array}{l}\text { I do not } \\
\text { agree }\end{array}$ & Undecided & I agree & $\begin{array}{l}\text { I strongly } \\
\text { agree }\end{array}$ & Mean & $\begin{array}{c}\text { Std. } \\
\text { Deviation }\end{array}$ \\
\hline \multirow{2}{*}{ Q 1} & Frequency & 19 & 19 & 24 & 76 & 176 & \multirow{2}{*}{4,18} & \multirow{2}{*}{1,181} \\
\hline & Percent & 6,1 & 6,1 & 7,6 & 24,2 & 56,1 & & \\
\hline \multirow{2}{*}{ Q 2} & Frequency & 15 & 18 & 55 & 94 & 132 & \multirow{2}{*}{3,99} & \multirow{2}{*}{1,122} \\
\hline & Percent & 4,8 & 5,7 & 17,5 & 29,9 & 42,0 & & \\
\hline \multirow{2}{*}{ Q 3} & Frequency & 14 & 8 & 14 & 74 & 204 & \multirow{2}{*}{4,42} & \multirow{2}{*}{1,012} \\
\hline & Percent & 4,5 & 2,5 & 4,5 & 23,6 & 65,0 & & \\
\hline \multirow{2}{*}{ Q4 } & Frequency & 9 & 11 & 20 & 75 & 199 & \multirow{2}{*}{4,41} & \multirow{2}{*}{ 963 } \\
\hline & Percent & 2,9 & 3,5 & 6,4 & 23,9 & 63,4 & & \\
\hline \multirow{2}{*}{ Q 5} & Frequency & 9 & 13 & 18 & 85 & 189 & \multirow{2}{*}{4,38} & \multirow{2}{*}{972} \\
\hline & Percent & 2,9 & 4,1 & 5,7 & 27,1 & 60,2 & & \\
\hline \multirow{2}{*}{ Q 6} & Frequency & 10 & 8 & 38 & 66 & 192 & \multirow{2}{*}{4,34} & \multirow{2}{*}{1,003} \\
\hline & Percent & 3,2 & 2,5 & 12,1 & 21,0 & 61,1 & & \\
\hline \multirow{2}{*}{ Q 7 } & Frequency & 18 & 15 & 38 & 96 & 147 & \multirow{2}{*}{4,08} & \multirow{2}{*}{1,138} \\
\hline & Percent & 5,7 & 4,8 & 12,1 & 30,6 & 46,8 & & \\
\hline \multirow{2}{*}{ Q 8} & Frequency & 16 & 18 & 44 & 93 & 143 & \multirow{2}{*}{4,05} & \multirow{2}{*}{1,134} \\
\hline & Percent & 5,1 & 5,7 & 14,0 & 29,6 & 45,5 & & \\
\hline \multirow{2}{*}{ Q9 } & Frequency & 9 & 15 & 34 & 93 & 163 & \multirow{2}{*}{4,23} & \multirow{2}{*}{1,013} \\
\hline & Percent & 2,9 & 4,8 & 10,8 & 29,6 & 51,9 & & \\
\hline \multirow{2}{*}{ Q 10} & Frequency & 13 & 26 & 40 & 94 & 141 & 00 & 1120 \\
\hline & Percent & 4,1 & 8,3 & 12,7 & 29,9 & 44,9 & 4,03 & 1,133 \\
\hline 11 & Frequency & 9 & 25 & 78 & 79 & 123 & 200 & 1100 \\
\hline Q 11 & Percent & 2,9 & 8,0 & 24,8 & 25,2 & 39,2 & 3,90 & 1,100 \\
\hline 019 & Frequency & 11 & 19 & 64 & 106 & 114 & 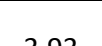 & 1050 \\
\hline Q 12 & Percent & 3,5 & 6,1 & 20,4 & 33,8 & 36,3 & 3,93 & 1,000 \\
\hline 012 & Frequency & 10 & 12 & 23 & 91 & 178 & 437 & ८९० \\
\hline Q 10 & Percent & 3,2 & 3,8 & 7,3 & 29,0 & 56,7 & 4,32 & (950 \\
\hline 14 & Frequency & 11 & 3 & 31 & 82 & 187 & 37 & 055 \\
\hline & Percent & 3,5 & 1,0 & 9,9 & 26,1 & 59,6 & & \\
\hline 15 & Frequency & 9 & 13 & 30 & 88 & 174 & 20 & 096 \\
\hline Q 10 & Percent & 2,9 & 4,1 & 9,6 & 28,0 & 55,4 & 4,29 & ,950 \\
\hline 016 & Frequency & 14 & 16 & 57 & 77 & 150 & 406 & 1125 \\
\hline & Percent & 4,5 & 5,1 & 18,2 & 24,5 & 47,8 & & \\
\hline
\end{tabular}

As can be seen from the table above; the statements concerning "organizational trust" are questions 3, 4 and 5 consequently. "I can count on my immediate supervisor for help if I have difficulties with my job." has a mean of 4.42, "I have complete trust that my immediate supervisor will treat me fairly." has a mean of 4.41, "I feel free to discuss work problems with my immediate supervisor." has a mean of 4.38 .

Likewise, the items that have the lowest scores are questions are 11, 12 and 2. "I can share sensitive information with members of my workgroup because I know group members will hold it in strict confidence." has a mean of 3.90, "The level of trust among 
people I work with on a regular basis is very high." has a mean of 3.93 and "If I make a mistake my supervisor is willing to "forgive and forget."'" has a mean of 3.99 .

Explanatory factor analysis of the leadership scale is as follows:

Table 4: Factor Analysis of the Leadership Scale

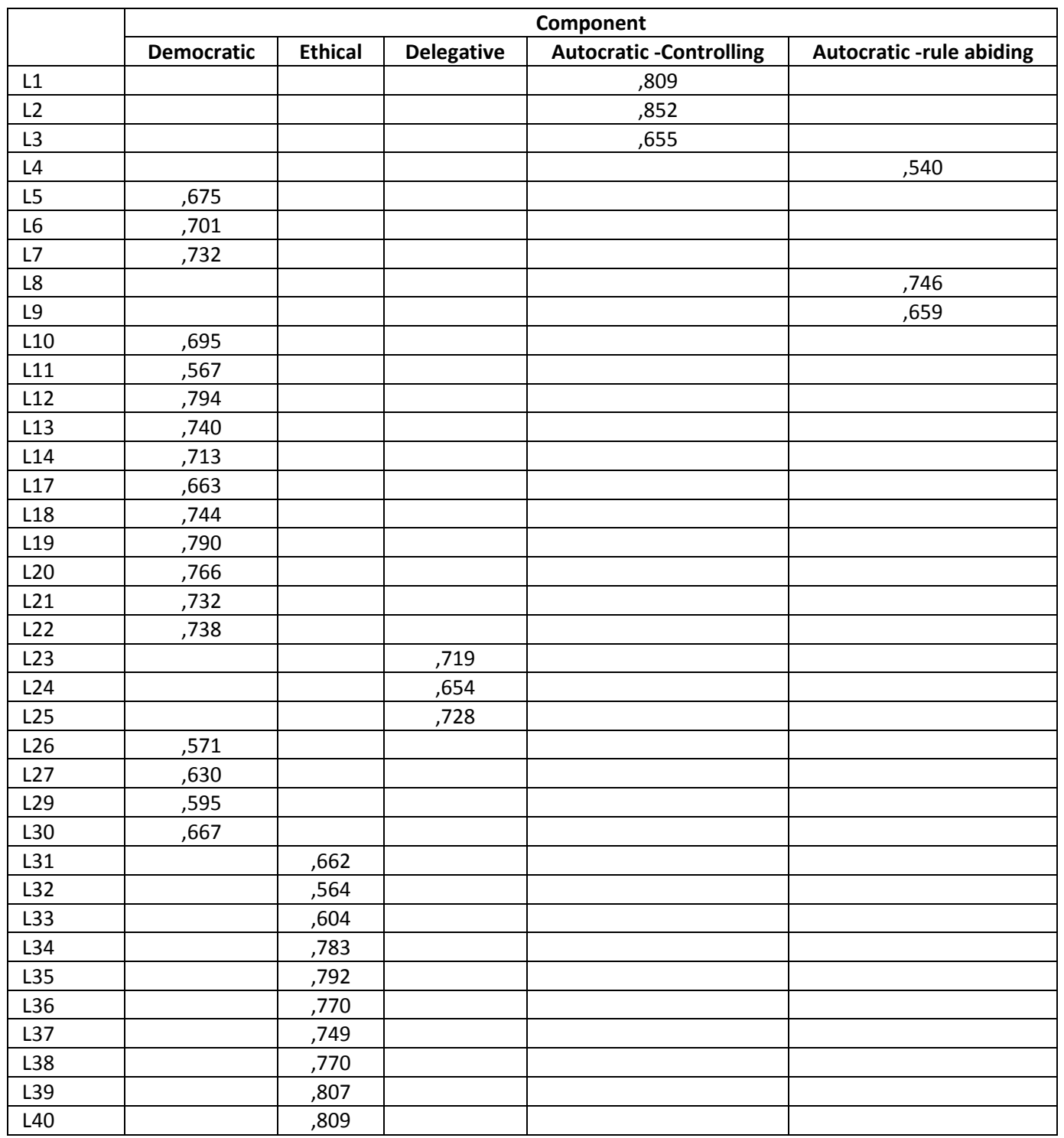

Three questions that are found to be below .50 (i.e., questions 15, 16 and 28 ) are omitted from the analysis. Democratic leadership component of the scale explained $44 \%$ of variance where the total variance was almost $65 \%$ (See Table 5 in the Appendix). 
Explanatory factor analysis of the organizational trust scale is as follows:

Table 5: Factor Analysis of Trust Scale

\begin{tabular}{|c|c|c|}
\hline & \multicolumn{2}{|c|}{ Component } \\
\hline & Managerial Trust & Interpersonal Trust \\
\hline Q 1 & 811 & \\
\hline Q2 & 653 & \\
\hline Q 3 & 810 & \\
\hline Q 4 & 778 & \\
\hline Q 5 & 834 & \\
\hline Q 6 & 771 & \\
\hline Q 7 & & ,780 \\
\hline Q 8 & & ,826 \\
\hline Q9 & & 654 \\
\hline Q 10 & & 855 \\
\hline Q 11 & & ,863 \\
\hline Q 12 & & ,594 \\
\hline Q 13 & 676 & \\
\hline Q 14 & ,735 & \\
\hline Q 15 & ,648 & \\
\hline Q 16 & & ,782 \\
\hline
\end{tabular}

Managerial trust component of the scale explained $64 \%$ of variance where the total variance was almost $73 \%$ (See Table 7 in the Appendix).

Internal consistency and reliability is high for both leadership (0.954) and trust (0.962). (See the Table 8 on Reliability/ Cronbach's Alpha in the Appendix). 
Figure 2: SEM Model 1 - The Effect of Trust on Leadership Styles

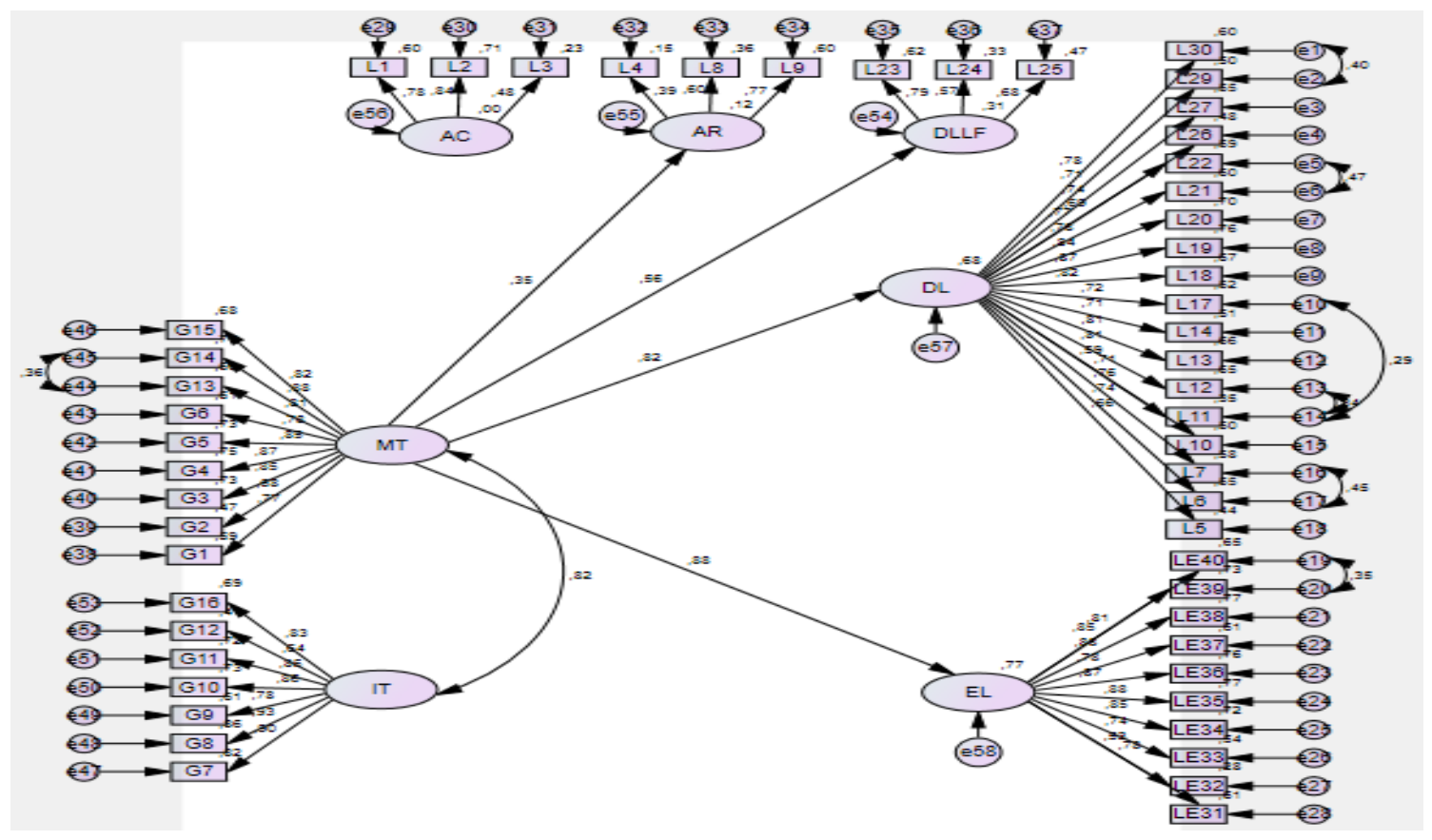

\begin{tabular}{cl|c|}
\hline Table 6: Structural Equation Model 1 & SEM Model 1 & \multicolumn{1}{c|}{$\mathbf{R}^{\mathbf{2}}$} \\
\hline DL/ LF & $=0,559^{*}(\mathrm{MT})$ & $\mathbf{0 , 3 1 2}$ \\
AC & $=0,353^{*}(\mathrm{MT})$ & $\mathbf{0 , 1 2 5}$ \\
EL & $=0,876^{*}(\mathrm{MT})$ & $\mathbf{0 , 7 6 8}$ \\
DL & $=0,824 *(\mathrm{MT})$ & $\mathbf{0 , 6 7 9}$ \\
\hline
\end{tabular}

(Goodness of fit: Cmin/df:2.365; GFI:0.726; CFI: 0.873; AGFI: 0.701; PGFI: 0.666; NFI: 0.8; TLI: 0.876). (Bootstrap:900 max likelihood).

The first SEM has been found significant at $5 \%$ confidence level. In this model, dependent variable is leadership style and the independent variable is organizational trust.

There is a positive causal relation between delegative leadership and managerial trust ( +0.559 standardized weight/ coefficient). Managerial trust has explained with an effect of $31.2 \%$ of delegative leadership style.

There is a positive causal relation between autocratic (controlling) leadership and managerial trust (+0.353 coefficient). Managerial trust has explained with an effect of $12.5 \%$ on autocratic (controlling) leadership style.

There is a positive causal relation between ethical leadership and managerial trust $(+0.876$ coefficient). Managerial trust has explained with an effect of $76.8 \%$ on ethical leadership style.

There is a positive causal relation between democratic leadership and managerial trust ( +0.824 coefficient). Managerial trust has explained with an effect of $67.9 \%$ on democratic leadership style. 
Figure 3: SEM Model 2 - The Effect of Leadership Styles on Trust

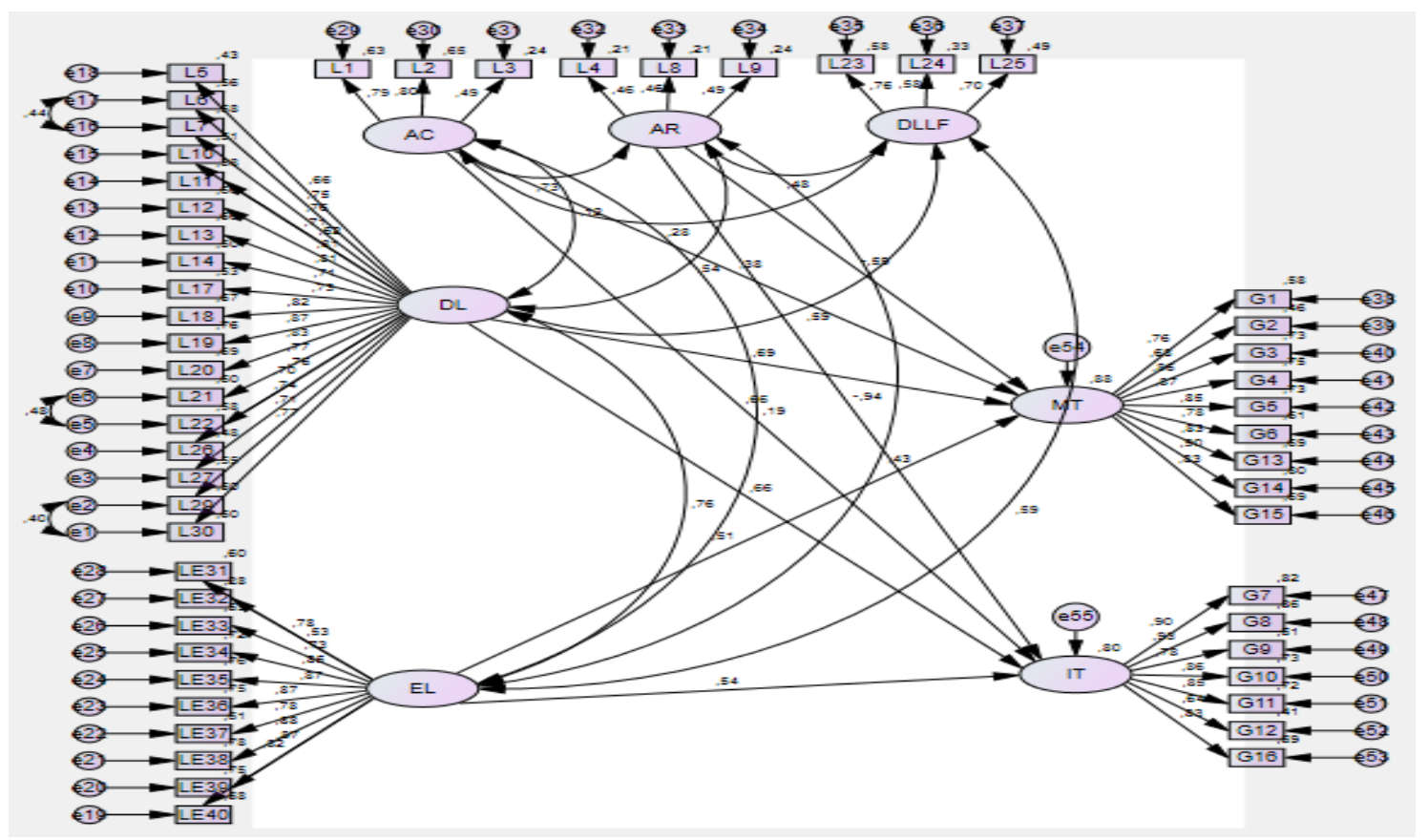

Figure 2: SEM MODEL 2: The effect of Leadership Styles on Trust

Table 7: Structural Equation Model 2

\begin{tabular}{ccc|c|}
\hline & SEM MODEL 2 & R2 \\
\hline IT & $=-0,942 * A R+0,661 * A C+0,536 * E L+0,655 * \mathrm{DL}$ & $\mathbf{0 , 8 0 3}$ \\
MT & $=-0,586 * A R+0,507 * \mathrm{EL}+0,692 * \mathrm{DL}$ & $\mathbf{0 , 8 7 7}$ \\
\hline
\end{tabular}

(Goodness of fit: Cmin/df:2.414; GFI:0.721; CFI: 0.869; AGFI: 0.694; PGFI: 0.657; NFI: 0.797; TLI: 0.862).(Not multivariate normality; Bootstrap:900 max.likelihood)

The second SEM has been found significant at $5 \%$ confidence level. In this model, dependent variable is organizational trust and the independent variable is leadership style.

There is a negative causal relation between interpersonal trust and autocratic (rule- abiding) leadership (-0.942 standardized value/ coefficient). Rest of the relations are all positive. There are positive causal relationships between interpersonal trust and autocratic (controlling) leadership ( 0.661 coefficient), ethical leadership ( 0.536 coefficient), democratic leadership ( 0.655 coefficient). Autocratic (rule - abiding), autocratic (controlling), ethical and democratic leadership styles, in other words, total leadership styles have explained with an effect of $80.3 \%$ of interpersonal trust.

There is a negative causal relation between managerial trust and autocratic (rule- abiding) leadership (-0.586 coefficient). Rest of the relations are all positive. There are positive causal relationships between managerial trust and ethical leadership $(0.507$ coefficient), democratic leadership (0.692 coefficient). Autocratic (rule - abiding), ethical and democratic leadership styles, in other words, total leadership styles have explained with an effect of $87.7 \%$ of managerial trust. On table 8 , the reliability (Cronbach's Alpha) scores and on Table 14 are correlations displayed. 
Table 8: Reliability

\begin{tabular}{|l|c|c|c|}
\hline \multicolumn{1}{|c|}{ Variables } & $\begin{array}{c}\text { Cronbach's } \\
\text { Alpha }\end{array}$ & $\begin{array}{c}\text { Cronbach's Alpha Based on } \\
\text { Standardized Items }\end{array}$ & N of Items \\
\hline Democratic Leadership &, 958 &, 960 & 18 \\
\hline Ethical Leadership &, 944 &, 946 & 10 \\
\hline Delegative Leadership &, 720 &, 722 & 3 \\
\hline Autocratic -Controlling &, 736 &, 731 & 3 \\
\hline Autocratic - Rule abiding &, 607 &, 617 & 3 \\
\hline Leadership Total &, 954 &, 959 & 37 \\
\hline Managerial Trust &, 946 &, 948 & 9 \\
\hline Interpersonal Trust &, 938 &, 938 & 7 \\
\hline $\begin{array}{l}\text { Organizational Trust } \\
\text { Total }\end{array}$ &, 962 &, 963 & 16 \\
\hline
\end{tabular}

Table 9 : Correlations Matrix

\begin{tabular}{|c|c|c|c|c|c|c|c|c|}
\hline \multicolumn{2}{|c|}{ Spearman's rho } & $\begin{array}{l}\text { Democratic } \\
\text { Leadership }\end{array}$ & $\begin{array}{c}\text { Ethical } \\
\text { Leadership }\end{array}$ & $\begin{array}{l}\text { Delegative } \\
\text { Leadership }\end{array}$ & $\begin{array}{l}\text { Autocratic- } \\
\text { Controlling }\end{array}$ & $\begin{array}{l}\text { Autocratic- } \\
\text { rule abiding }\end{array}$ & $\begin{array}{c}\text { Manager } \\
\text { ial Trust }\end{array}$ & $\begin{array}{c}\text { Interpersonal } \\
\text { Trust }\end{array}$ \\
\hline \multirow[t]{3}{*}{$\begin{array}{l}\text { Democratic } \\
\text { Leadership }\end{array}$} & $\begin{array}{l}\text { Correlation } \\
\text { Coefficient }\end{array}$ & 1,000 &, $746^{* *}$ &, $511^{* *}$ & $143^{*}$ &, $254^{* *}$ &, $730^{* *}$ &, $606^{* *}$ \\
\hline & Sig. (2-tailed) & & ,000 & ,000 & 011 & ,000 & ,000 & ,000 \\
\hline & $\mathrm{N}$ & 314 & 314 & 314 & 314 & 314 & 314 & 314 \\
\hline \multirow[t]{3}{*}{$\begin{array}{l}\text { Ethical } \\
\text { Leadership }\end{array}$} & $\begin{array}{l}\text { Correlation } \\
\text { Coefficient }\end{array}$ & ,746** & 1,000 &, $484^{* *}$ & ,189** &, $253^{* *}$ & ,766** &, $661^{* *}$ \\
\hline & Sig. (2-tailed) & ,000 & & ,000 & ,001 & ,000 & ,000 & ,000 \\
\hline & $\mathrm{N}$ & 314 & 314 & 314 & 314 & 314 & 314 & 314 \\
\hline \multirow[t]{3}{*}{$\begin{array}{l}\text { Delegative } \\
\text { Leadership }\end{array}$} & $\begin{array}{l}\text { Correlation } \\
\text { Coefficient }\end{array}$ &, $511^{* *}$ &, $484^{* *}$ & 1,000 &, $218^{* *}$ & ,299** &, $432^{* *}$ & , 380** \\
\hline & Sig. (2-tailed) & 000 & ,000 & & ,000 & ,000 & ,000 & ,000 \\
\hline & $\mathrm{N}$ & 314 & 314 & 314 & 314 & 314 & 314 & 314 \\
\hline \multirow[t]{3}{*}{$\begin{array}{l}\text { Autocratic } \\
\text { Controlling }\end{array}$} & $\begin{array}{l}\text { Correlation } \\
\text { Coefficient }\end{array}$ &, $143^{*}$ &, $189^{* *}$ &, $218^{* *}$ & 1,000 &, $410^{* *}$ &, $111^{*}$ &, $173^{* *}$ \\
\hline & Sig. (2-tailed) & 011 & ,001 & ,000 & & ,000 & ,050 & ,002 \\
\hline & $\mathrm{N}$ & 314 & 314 & 314 & 314 & 314 & 314 & 314 \\
\hline \multirow[t]{3}{*}{$\begin{array}{l}\text { Autocratic } \\
\text { Rule abiding }\end{array}$} & $\begin{array}{l}\text { Correlation } \\
\text { Coefficient }\end{array}$ &, $254^{* *}$ &, $253^{* *}$ & ,299** &, $410^{* *}$ & 1,000 & $131^{*}$ & 110 \\
\hline & Sig. (2-tailed) & ,000 & ,000 & ,000 & ,000 & & 021 & ,052 \\
\hline & $\mathrm{N}$ & 314 & 314 & 314 & 314 & 314 & 314 & 314 \\
\hline \multirow[t]{3}{*}{$\begin{array}{l}\text { Managerial } \\
\text { Trust }\end{array}$} & $\begin{array}{l}\text { Correlation } \\
\text { Coefficient }\end{array}$ & ,730** &, $766^{* *}$ &, $432^{* *}$ & $111^{*}$ &, $131^{*}$ & 1,000 &, $755^{* *}$ \\
\hline & Sig. (2-tailed) & ,000 & ,000 & ,000 & ,050 & ,021 & & ,000 \\
\hline & $\mathrm{N}$ & 314 & 314 & 314 & 314 & 314 & 314 & 314 \\
\hline \multirow[t]{3}{*}{$\begin{array}{l}\text { Interpersonal } \\
\text { Trust }\end{array}$} & $\begin{array}{l}\text { Correlation } \\
\text { Coefficient }\end{array}$ &, $606^{* *}$ &, $661^{* *}$ &, $380^{* *}$ &, $173^{* *}$ & 110 &, $755^{* *}$ & 1,000 \\
\hline & Sig. (2-tailed) & ,000 & ,000 & ,000 & ,002 & ,052 & ,000 & \\
\hline & $\mathrm{N}$ & 314 & 314 & 314 & 314 & 314 & 314 & 314 \\
\hline
\end{tabular}

**. Correlation is significant at the 0.01 level (2-tailed). *. Correlation is significant at the 0.05 level (2-tailed). 


\begin{tabular}{|c|c|c|c|c|c|}
\hline Dependent Variable & Indepe & dent Variable & \multirow{2}{*}{$\mathbf{Z}$} & \multirow{2}{*}{ Sig. } & \multirow{2}{*}{ Explanation } \\
\hline \multirow{9}{*}{ Delegative leadership } & \multicolumn{2}{|c|}{ Hometown } & & & \\
\hline & Village & Town/district & \multirow{2}{*}{$-2,278$} & \multirow{2}{*}{,023 } & \multirow{8}{*}{$\begin{array}{l}\text { There is significant } \\
\text { difference between } \\
\text { the teachers whose } \\
\text { hometowns are } \\
\text { villages and small } \\
\text { towns versus the } \\
\text { others. The first } \\
\text { perceives more } \\
\text { delegative leadership } \\
\text { style than the latter. }\end{array}$} \\
\hline & 69,33 & 53,63 & & & \\
\hline & Sub -district & Town/District & \multirow[t]{2}{*}{$-2,681$} & \multirow[t]{2}{*}{,007 } & \\
\hline & 67,50 & 46,25 & & & \\
\hline & Sub -district & Province & \multirow{2}{*}{$-2,390$} & \multirow{2}{*}{,017 } & \\
\hline & 54,70 & 38,54 & & & \\
\hline & Sub -district & Metropolitan City & \multirow{2}{*}{$-1,958$} & \multirow{2}{*}{,050 } & \\
\hline & 84,57 & 64,18 & & & \\
\hline \multirow{8}{*}{ Delegative leadership } & \multicolumn{2}{|r|}{ Age } & & & \multirow{8}{*}{$\begin{array}{l}\text { Older teachers are } \\
\text { perceiving their } \\
\text { principals' styles as } \\
\text { more delegative than } \\
\text { the others. }\end{array}$} \\
\hline & 20-29 age & $30-39$ age & & & \\
\hline & 132,56 & 157,54 & $-2,5<3$ & , & \\
\hline & $20-29$ age & 50 years and over & & & \\
\hline & 87,07 & 137,50 & & & \\
\hline & 30-39 age & 50 years and over & & & \\
\hline & & & $-1,959$ & ,050 & \\
\hline & 58,21 & 88,60 & & & \\
\hline \multirow{9}{*}{ Democratic leadership } & \multicolumn{2}{|r|}{ Age } & & & \multirow{9}{*}{$\begin{array}{l}\text { Older teachers of age } \\
50 \text { years and over are } \\
\text { perceiving their } \\
\text { principals' styles as } \\
\text { more democratic than } \\
\text { the others. }\end{array}$} \\
\hline & $20-29$ age & 50 years and over & & & \\
\hline & & & $-2,791$ & ,005 & \\
\hline & 86,67 & 151,10 & & & \\
\hline & 30-39 age & 50 years and over & & & \\
\hline & 57,82 & 97,50 & & & \\
\hline & $40-49$ age & 50 years and over & \multirow{3}{*}{$-2,025$} & \multirow{3}{*}{,043 } & \\
\hline & & & & & \\
\hline & 14,06 & 22,70 & & & \\
\hline \multirow{9}{*}{ Ethical leadership } & & Age & & & \multirow{9}{*}{$\begin{array}{l}\text { Older teachers of age } \\
50 \text { years and over are } \\
\text { perceiving their } \\
\text { principals' styles as } \\
\text { more ethical than the } \\
\text { others. }\end{array}$} \\
\hline & $20-29$ age & 50 years and over & \multirow{3}{*}{$-2,537$} & \multirow{3}{*}{011} & \\
\hline & & & & & \\
\hline & 86,84 & 145,30 & & & \\
\hline & 30-39 age & 50 years and over & \multirow{2}{*}{$-2,342$} & \multirow{2}{*}{019} & \\
\hline & 57,96 & 94,40 & & & \\
\hline & 40-49 age & 50 years and over & & & \\
\hline & & & $-2,379$ & 017, & \\
\hline & 13,80 & 24,00 & & & \\
\hline
\end{tabular}




\begin{tabular}{|c|c|c|c|c|c|}
\hline $\begin{array}{l}\text { P.S: There is no significant } \\
\text { relation }\end{array}$ & $\begin{array}{r}\text { between at } \\
d\end{array}$ & $\begin{array}{l}\text { cratic leadership \& } \\
\text { ography. }\end{array}$ & & & \\
\hline \multirow{9}{*}{ Interpersonal Trust } & & Age & & & \multirow{9}{*}{$\begin{array}{l}\text { Older teachers of age } \\
50 \text { years and over } \\
\text { have higher } \\
\text { interpersonal trust } \\
\text { than the others. }\end{array}$} \\
\hline & 20-29 age & 50 years and over & \multirow{3}{*}{$-2,649$} & \multirow{3}{*}{,008 } & \\
\hline & & & & & \\
\hline & 86,77 & 147,60 & & & \\
\hline & 30-39 age & 50 years and over & \multirow{2}{*}{$-2,526$} & \multirow{2}{*}{,012 } & \\
\hline & 57,84 & 97,00 & & & \\
\hline & 40-49 age & 50 years and over & \multirow{3}{*}{$-2,504$} & \multirow{3}{*}{,012 } & \\
\hline & & & & & \\
\hline & 13,72 & 24,40 & & & \\
\hline
\end{tabular}

Out of five demographic variables (gender, education, seniority, marital status, hometown, and age, only the latter two variables have been found significantly meaningful.

\section{CONCLUSION}

We have limited our sample to secular state schools where principals are appointed rather than selected. Meanwhile, our survey has been distributed and collected by principals and vice principals; that is why, teachers have felt somewhat uneasy. This might have effected their anwers. Autocratic leadership has been broken down into two components: rule-abiding and bureaucratic versus controlling styles. The first one has been found significant, while the latter has insignificant paths as independent variables. This might be as a result of the representative nature of principals of the education system in state schools. It would be more appropriate to include private junior high schools (primary and secondary) in future research.

Moreover, autocratic rule-abiding leadership style has high negative effect on both managerial and interpersonal trust. Rigid rules and high expectations of compliance based on "procedural justice" without taking specific context into account might have led to negative results such as decreasing motivation of teachers. However, autocratic controlling leadership has positive effect on interpersonal trust which may be interpreted as a perception of "distributive or restorative justice" among colleagues.

As an emergent economy, education system is continually changing both its programs as well as its structures. Therefore, principals have difficulty in adapting and internalizing new priorities and expectations. Delegative leadership style has become more predominant as a consequence of hands-off policy often followed by principals at times of uncertainty and complexity. Further studies may be done concerning the two cultural dimensions such as power distance, uncertainty avoidance which are particularly high (Hofstedte; 1980).

Only managerial trust has a significant effect on leadership styles which reveals the fact that dyadic relationships between leaders and employees enhance mutual trust. Managerial trust has high explanatory power on ethical and democratic leadership styles as compared to the other styles, since they both have higher degrees of interaction. We have not employed the systemic level of analysis i.e., organizational level which reduces complexity that Luhmann had suggested in his definition of confidence and communication, since he regards them both essential for coordination within and among system at large. Future studies may take into account the organizational trust separately.

On the whole, Csikszentmihalyi's four major leadership competencies that are directly linked to his notion of flow are also found to be significant and relevant to managerial trust as well as ethical leadership as we have anticipated: (1) Strategic thinking (e.g., setting clear meaningful goals), (2) Applying personal strengths for a common goal (such as self-confidence, interpersonal communication), (3) Balancing skill and challenge level (e.g., focusing on competencies and efforts, that is, labor along with results), and (4) Frequent feedback and encouragement on performance and interpersonal relations.

In future studies on primary and secondary schools (junior high schools) in the metropolitan city (Istanbul), a larger sample size is needed to be able to generalize the findings to the population at large. Moreover, a comparison between secular as opposed to religious schools as well as public contrasted with private schools may be queried. However, Istanbul is representative of the diversity of the country and the appointed teachers are from diverse socioeconomic backgrounds, and the students of the district we have chosen are mostly from migrant families within Turkey. 


\section{REFERENCES}

Adams, S. (2004). The Relationships Among Adult Attachment, General Self-Disclosure, and Perceived Organizational Trust: Dissertation submitted to the faculty of the Virginia Polytechnic Institute and State University In partial fulfillment of the requirements for the degree of, Doctor of Philosophy in Human Development.

Ateş, G. (2005). Yöneticilerin Liderlik Tarzlarının Çalışanların İş Tatmini Üzerindeki etkileri: Hava Kuvvetleri Komutanlığında Uygulama. Kütahya: Dumlupınar Üniversitesi Sosyal Bilimler Enstitüsü Basılmamış Yüksek Lisans Tezi (unpublished graduate thesis).

Barber, B. (1983). The Logic and Limits of Trust. New Jersey: Rutgers University Press.

Begeç, S. (1999). Modern Liderlik Yaklaşımları ve Uygulaması. Gebze Yüksek Teknoloji Enstitüsü, Mühendislik ve Fen Bilimleri Enstitüsü.

Bhatti, N., Maitlo, G. M., Shaikh, N., Hashmi, M. A., \& Shaikh, F. M. (2012, February). The Impact of Autocratic and Democratic Leadership Style on Job Satisfaction. International Business Research, 5(2), 192-201. www.ccsenet.org/ibr (09.02.2019).

Blomqvist, K., \& Ståhle, P. (1998). Building Organizational Trust. 1-16.

Brown, M., \& Trevino, L. (2014). Do Role Models Matter? An Investigation of Role Modeling as an Antecedent of Perceived Ethical Leadership. Journal Bus Ethics, 122, 587-598.

Buch, R., Martinsen, Ø. L., \& Kuvaas, B. (2015). The Destructiveness of Laissez-Faire Leadership Behavior: The Mediating Role of Economic LeaderMember Exchange Relationships. Journal of Leadership \& Organizational Studies, 22(11), 115-124.

Clark, D. C., \& Clark, S. N. (1997). Addressing Dilemmas Inherent in Educational Leadership Preparation Programs Through Collaborative Restructuring. Peabody Journal of Education, 72(2), 21-41.

Clark, S., \& Clark, D. (2003). Perspectives on Three Decades of the Middle Level Principalship. Middle School Leadership, 35(2), 48-54.

Clark, S., \& Clark, D. (2004). Expert Leadership and Comprehensive Professional Development: A Key to Quality Educators in Middle Schools. Middle School Journal, 35(4), 47-53.

Csikszentmihalyi, Mihaly (1990). Flow: The Psychology of Optimal Experience. New York: Harper and Row.

Hofstedte, G. (1980). Cultures and Organizations: Software of the Mind, N.Y.: McGraw-Hill.

Jalava, J. (2006). Trust as a Decision: The Problems and Functions of Trust in Luhmannian Systems Theory. Helsinki: University of Helsinki, Faculty of Social Sciences,Department of Social Policy, Research Reports.

Retrieved from http://ethesis.helsinki.fi/julkaisut/val/sospo/vk/jalava/trustasa.pdf (09.02.2018).

Kanten, P. (2012). İş Görenlerde İşe Adanmanın ve Proaktif Davranışların Oluşumunda Örgütsel Güven ile Örgütsel Özdeşleşmenin Rolü. Isparta: T.C. Süleyman Demirel Üniversitesi Sosyal Bilimler Enstitüsü İşletme Anabilim Dalı Basılmamış Yüksek Lisans Doktora Tezi. (unpublished graduate thesis).

Lewin, Lippitt \& White (1939). Patterns of Aggressive Behavior in Experimentally Created "Social Climates". Journal of Social Psychology, 10(2), 271-299.

Luhmann, N. (1979). Trust and Power. John Wiley \& Sons.

Luhmann, N. (2000). Trust: Making and Breaking Cooperative Relations. G. Diego içinde, Familiarity, Confidence, Trust: Problems and Alternatives (p. 94-107). Oxford: University of Oxford.

Mayer, R. C., Davis, J. H., \& Schoorman, D. F. (1995). An Integrative Model of Organizational Trust. Academy ol Management Review, 20(3), 709734.

McAllister, D. J. (1995). Affect and Cognition Based Trust as Foundation for Interpersonal Cooperation in Organizations. Academy of Management Journal, 38(1), 24-59.

Oshagbemi, T. and Gill, R. (2003). Differences in Leadership Styles and Behavior Across Hierarchical Levels in UK Organizations. Leadership Styles and Behaior. 25(1), 93-106.

Pawar, D. V. (2014). Styles of Leadership. International Journal of Research in all Subjects in Multi Languages, 2(7), 12.

Rast, D. E., Hogg, M. A., \& Giessner, S. R. (2013). Self-uncertainty and Support for Autocratic Leadership. Self and Identity, 12(6), 635-649.

Resick, C. J., Hargis, M. B., Shao, P., \& Dust, S. B. (2013). Ethical Leadership, Moral Equity Judgments, and Discretionary Workplace Behavior. Human Relations, 66(7), 951-972.

Scott, D., \& Cook, B. (1983). The Relationship Between Employee Age and Interpersonal Trust Within an Organizational Context. School of Business: Faculty Publications and Other Works, 69-83. 
Seppanen, R., Blomqvist, K., \& Sundqvist, S. (2007). Measuring Inter-Organizational Trust-A Critical Review of the Empirical Research in 19902003. Industrial Marketing Management, 36, 249 - 265.

Tuna, M., Bircan, H., \& Yeşiltaş, M. (2012). Etik Liderlik Ölçeğinin Geçerlilik ve Güvenilirlik Çalışması: Antalya Örneği. Atatürk Üniversitesi Iktisadi ve Idari Bilimler Dergisi, 26(2), 143-155.

Vidotto, G., Vicentini, M., Argentero, P., \& Bromiley, P. (2008). Assessment of Organizational Trust: Italian Adaptation and Factorial Validity of the Organizational Trust Inventory. Social Indicators Research Springer, 88, 563-575. https://link.springer.com/journal/11205 (06.09.2018). 